\title{
Variante anatómica sintomática de la duplicación de la vía biliar común tipo IV
}

\author{
Anatomy variant symptomatic of the duplication of the common bile duct type IV
}

Ana Alberca-Páramo ${ }^{*}$, Esther P. García-Santos ${ }^{2}$, David Padilla-Valverde $^{2}$, Pedro Villarejo-Campos ${ }^{3}$,
Mercedes Estaire-Gómez ${ }^{2}$ y Jesús Martín Fernández ${ }^{2}$
'Servicio Cirugía General y Aparato Digestivo, Hospital Comarcal San Agustín, Linares; ${ }^{2}$ Servicio Cirugía General y Aparato Digestivo, Hospital
General Universitario de Ciudad Real, Ciudad Real; ${ }^{3}$ Servicio Cirugía General y Aparato Digestivo, Hospital Fundación Jiménez Díaz, Madrid. España

\section{Resumen}

Introducción: La duplicidad del conducto biliar común (DCBC) es una alteración congénita insólita. Caso clínico: Mujer de 80 años con duplicación de la vía biliar común con colangiopancreatografía retrógrada endoscópica (CPRE) que no dilucida la clínica. Discusión: Este caso es una variante del tipo IV de la clasificación de DCBC. La colangiorresonancia y la CPRE prequirúrgica permiten valorar las vías biliares, su calibre y las anormalidades. El tratamiento depende de la clínica y el tipo de apertura del conducto biliar común accesorio. Conclusiones: Es importante realizar estudio prequirúrgico y durante la operación con colangiografía intraoperatoria.

PALABRAS CLAVE: Duplicación. Conducto biliar común. Conducto biliar común accesorio.

\begin{abstract}
Introduction: Duplicity of the common bile duct (BCBD) is an unusual congenital disorder. Case report: $A$ 80-year-old woman with duplication of the common bile duct with retrograde endoscopic cholangiopancreatography (ERCP) who did not resolve the symptoms. Discussion: Our case is a variant of type IV to the classification of DCBC. The MR cholangiography and presurgical ERCP allows assessment of the bile ducts, their caliber, and assessment of abnormalities. The treatment before $D C B C$ will depend on the clinic and the type of opening of the CBCA. Conclusions: It is important to perform a pre-surgical study and during surgery with $\mathrm{ClO}$.
\end{abstract}

KEY WORDS: Duplication. Common bile duct. Acessory common bile duct.

\section{Introducción}

La cirugía biliar es una de las más complejas desde el punto de vista estructural y posee múltiples variaciones anatómicas, que pueden conllevar complicaciones muy importantes'.
La duplicidad del conducto biliar común (DCBC), en el cual existen dos conductos biliares comunes, es una alteración congénita excepcional². Según la clasificación de Choi, et al., el tipo IV tiene dos vías biliares comunes, una que desemboca en el duodeno y la otra en distintos segmentos del tracto gastrointestinal superior, entre ellos el estómago, el 
duodeno o el conducto pancreático principal (éste recibe el nombre de conducto biliar común accesorio, $\mathrm{CBCA})^{2,3}$.

Esta anormalidad anatómica es de gran importancia porque estos pacientes presentan a menudo afección secundaria a esta alteración, entre las cuales destacan la litiasis en la vía biliar ectópica o los quistes del colédoco ${ }^{4}$. También puede relacionarse con anomalías de la unión pancreaticobiliar y neoplasias en el tracto gastrointestinal superior ${ }^{2}$.

La prueba diagnóstica prequirúrgica por excelencia para la valoración anatómica de la vía biliar es la colangiorresonancia magnética (CRM $)^{5}$.

Durante la operación, la mejor prueba para la detección de las variaciones anatómicas es la colangiografía intraoperatoria (CIO), que además permite valorar el tipo de opción quirúrgica a realizar y la detección temprana de posibles lesiones quirúrgicas ${ }^{6}$.

\section{Material y método}

Se trata de la exposición de un caso clínico inédito, una duplicación de la vía biliar con una variante no descrita antes en la bibliografía.

\section{Caso clínico}

Mujer de 80 años de edad con antecedentes personales de hipertensión, dislipemia, diabetes tipo 2 y síndrome depresivo en tratamiento. La paciente se derivó al Servicio de Aparato Digestivo por aumento de las transaminasas: glutámico-oxalacética (GOT) de $49 \mathrm{UI} / \mathrm{l}$, glutámico pirúvica (GPT) de $71 \mathrm{UI} / \mathrm{l}$ y otras enzimas como gammaglutamil transferasa (GGT) de $707 \mathrm{UI} / \mathrm{l}$ y fosfatasa alcalina (ALP) de $384 \mathrm{UI} / \mathrm{l}$, además de clínica de astenia, anorexia de meses de evolución y pérdida de peso no cuantificada. Asimismo, refería coluria dudosa y erupciones pruriginosas resueltas con tratamiento antihistamínico. Ante dicha situación se solicitó tomografía computarizada (TC) abdominal y un estudio analítico completo.

En la analítica se documentaron bilirrubina $(0,8 \mathrm{UI} / \mathrm{l})$, AST (70 UI/I), GGT (1.287 UI/I) y ALP (537 UI/I). El marcador CA19.9 se mantuvo en límites de normalidad y los anticuerpos ANA resultaron negativos, así como la serología vírica.

En la TC se reconoció una lesión hipodensa de $\sim 12 \mathrm{~mm}$ con morfología quística en el segmento III. La vesícula biliar se presentaba sin alteraciones morfológicas y se delineaba una masa hipercaptante en el colédoco distal de $\sim 20 \mathrm{~mm}$ de diámetro que provocaba una dilatación retrógrada de toda la vía biliar intrahepática y extrahepática. La vía biliar común, distal a la lesión descrita con anterioridad, y el Wirsung se encontraban levemente dilatados. La cabeza pancreática parecía un poco aumentada de tamaño, aunque no se identificaban lesiones en su parénquima.

El estudio se completó con una CRM, en la cual se observaba la existencia de una malformación o una divergencia de la normalidad de las vías biliares extrahepáticas consistente en una duplicación de la vía biliar común, con una desembocadura anómala de la vía biliar común duplicada. La vía biliar derecha hepática se continuaría con la vía biliar común, mientras que la izquierda, aunque se comunica con la derecha en la bifurcación, se continuaría con el colédoco duplicado que desembocaría de modo ectópico en el margen posterior y craneal del antro pilórico.

Se realizaron diferentes colangiopancreatografías retrógradas endoscópicas (CPRE) en las que se identificaron defectos de repleción, tanto en la vía biliar común normal como en el ectópico y en las primeras porciones de sus conductos derecho, izquierdo y la bifurcación de ambos.

Durante las distintas CPRE se efectuaron una esfinterotomía biliar amplia y múltiples colocaciones y remociones de endoprótesis plásticas de $10 \mathrm{Fr}$ y $7 \mathrm{~cm}$ de longitud.

Antes de la última CPRE se obtuvo TC abdominal de control en la cual se delineó una vesícula biliar distendida, de paredes lisas, sin signos de colecistitis y con ectasia de la vía biliar extrahepática e intrahepática central y extensa aerobilia izquierda en relación con la prótesis plástica en colédoco, en posición normal. Por ello se decidió en la CPRE retirar la endoprótesis y extraer barro biliar con balón de Fogarty. Con posterioridad se instituyó tratamiento domiciliario con ácido ursodesoxicólico cada ocho horas.

Cinco meses después, la paciente mostró otra vez signos clínicos biliares; se realizó una nueva CRM y se identificó una vía biliar común en posición anatómica normal hasta de $10 \mathrm{~mm}$ de diámetro y con claros defectos de repleción en su interior, un cístico y los segmentos posteriores hepáticos que drenan a través de esta vía y un segundo sistema que a partir de dos vías biliares hepáticas drena los segmentos anteriores del hígado; éstos se unían en una vía biliar común accesoria dilatada de $12 \mathrm{~mm}$ que drenaba al estómago en la zona posterosuperior del antro a 1-2 cm del píloro y que mostraba una imagen de litiasis distal de $8 \mathrm{~mm}$. La vía biliar común derecha 


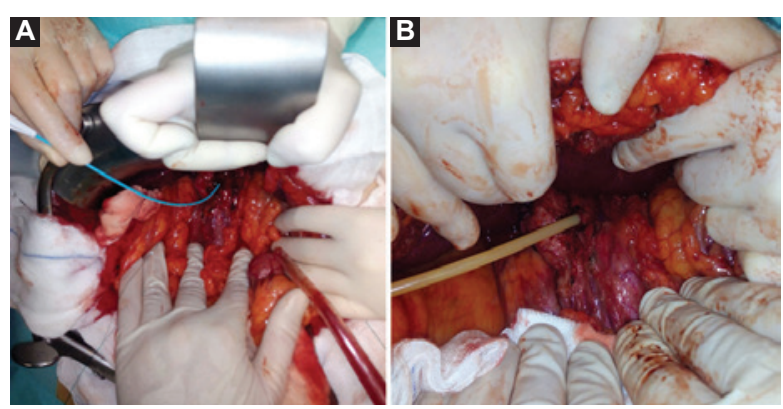

Figura 1. Tiempos quirúrgicos. A: colocación de catéter de colangiografía en $C B C A$. B: colocación de tubo en T en la CBCA fijado con poliglicólico 3-0.

del sistema accesorio y la común izquierda del normal contactaban de forma estrecha y parecían estar comunicadas; se reconoció una imagen de defecto de repleción en la zona de fusión consistente con litiasis de $14 \mathrm{~mm}$ de diámetro que se alojaba sobre todo en el sistema accesorio pero que obstruía de forma notable el paso a ramas posteriores izquierdas (sistema principal).

Tras dichos hallazgos, la paciente se remitió a consulta de Cirugía General donde se indicaron exploración de vía biliar y colangiografía intraoperatoria.

Mediante laparotomía subcostal derecha se exploraron las vías biliares y se observaron una vía biliar en posición normal y una vía biliar accesoria paralela a la desembocadura a nivel prepilórico. La colecistectomía reglada y la colangiografía intraoperatoria a través del cístico (Fig. 1A) reconocen el relleno de ambas vías con defectos de repleción en su interior, un signo indicativo de litiasis. Dado que no se delinea la desembocadura distal de la vía accesoria con claridad, se decide practicar una coledocotomía de esta vía, lavado con Fogarty que extrae múltiples cálculos y barro biliar, y la exploración de la vía biliar que hace posible visualizar la comunicación con el antro gástrico. Se coloca tubo en T (Fig. 1B) con sutura de poliglicólico 3-0. A continuación se solicita una nueva colangiografía y se comprueba el relleno hacia el estómago en dirección distal y relleno de contraste hacia el resto del árbol biliar, incluida la vía biliar principal en sentido proximal (Figs. 2A y B).

El postoperatorio transcurrió sin incidencias y recibió el alta el séptimo día de ingreso con buena tolerancia al tubo en $\mathrm{T}$ pinzado (Fig. $2 \mathrm{C}$ ). La anatomía patológica informó que se trataba de una vesícula con componente de colecistitis crónica, sin ninguna alteración tumoral. La paciente se encuentra hoy en día asintomática.

\section{Discusión}

La anatomía habitual del árbol biliar se encuentra en el $58 \%$ de la población y se presentan anomalías en el $42 \%$ restante $^{3}$. Las alteraciones más frecuentes se encuentran en la vía biliar intrahepática como el drenaje aberrante de la vía biliar posterior derecha en la vía biliar hepática izquierda y el drenaje simultáneo de la posterior derecha, la anterior derecha y la hepática izquierda a la vía biliar hepática común ${ }^{7,8}$.

Sin embargo, las duplicaciones de las vías biliares extrahepáticas son una de las variantes anatómicas más inusual, con alrededor de 130 casos publicados en la bibliografía ${ }^{3,8}$. En la actualidad se desconoce la incidencia real; quizás sea mucho más alta de lo previsto ${ }^{9}$.

Vesalio descubrió por primera vez esta anomalía en $1543^{3,10}$. Teilum identificó en 1986 una serie de 24 casos en el mundo occidental2; Kanematsu, et al. revisaron 56 casos en 1992 en todo el mundo occidental; Yamashita revisó en las publicaciones japonesas desde 1968 hasta 2002 y recopiló una serie de 47 pacientes con dicha anomalía ${ }^{10}$ y Gengchen, et al. revisaron 24 casos en la bibliografía china ${ }^{3}$.

El desarrollo embrionario de la vesícula biliar, el hígado y el sistema biliar tiene lugar hacia la tercera semana de gestación ${ }^{3}$, cuando el divertículo hepático, que es el resultado de la evolución de la endodermis distal del intestino anterior, comienza a crecer y sus células penetran en el mesénquima del mesogastrio ventral y se divide en una yema ventral y otra dorsal. La vesícula biliar se forma a partir de esta yema ventral y el brote dorsal se divide en los lóbulos derecho e izquierdo. El desarrollo de hígado y árbol biliar se realiza de forma conjunta ${ }^{2,11}$. El conducto biliar surge del tallo primitivo hepático y su unión con el brote ventral forma el cístico ${ }^{9}$. Es importante recalcar que la rotación del duodeno primitivo $90^{\circ}$ a lo largo de su eje hace que la vía biliar se eleve hacia el duodeno $0^{2,3,8}$.

El origen de esta anomalía anatómica podría relacionarse con la subdivisión aleatoria del divertículo hepático durante la primera semana de la embriogénesis ${ }^{2,12}$. La DCBC podría deberse a alteraciones en la recanalización del primordio hepático ${ }^{12}$.

EI CBCA no se abre a la papila duodenal principal; se suele abrir en diferentes partes del tracto gastrointestinal superior como son todas las porciones del duodeno, incluida la zona justo por encima de la papila duodenal mayor, al conducto pancreático 
principal ${ }^{2}$, o por medio de un tabique en el conducto biliar común? .

Existe una clasificación basada en la anatomía de los distintos tipos de la DCBC que realizaron en 1972 Goor y Ebert $^{13}$, la cual modificaron en 1988 Saito, et al. ${ }^{14}$ y posteriormente en 2007 Choi, et al., según la cual hay cinco tipos de DCBC en función de la morfología ${ }^{2}$ (Fig. $\left.3 A, B\right)$. Tipo I: existe un tabique que divide el conducto biliar; tipo II: presenta una bifurcación del conducto distal con un drenaje intestinal independiente; tipo III: existencia de duplicaciones de las vías biliares extrahepáticas sin (a) 0 con los canales de comunicación intrahepáticas (b); tipo IV: existencia de duplicación de las vías biliares extrahepáticas; y tipo V: presencia de drenaje biliar a conductos biliares $\sin$ (a) o con canales comunicantes $(b)^{3,8,15}$.

Los subtipos más frecuentes son III o IV ${ }^{8}$ y los menos comunes el $\mathrm{V}$ con un solo caso de cada tipo: Va en Choi, et al..$^{15}$ y Vb en Kim. et al., $2008^{8}$.

Según las revisiones encontradas en la bibliografía, dicha anomalía es más frecuente en Asia, sobre todo en Japón ${ }^{3}$, por lo que podría tener relación con el origen étnico ${ }^{3}$.

Las complicaciones más frecuentes de CBCA suelen ser coledocolitiasis, colangitis, pancreatitis, cáncer gástrico o de las vías biliares, quistes del colédoco, anomalías de la unión pancreaticobiliar como atresia ${ }^{2,7,8}$. El cáncer gástrico es una de las complicaciones que pueden objetivarse en los pacientes con CBCA que drena en el estómago, en el tipo III, tal y como el cáncer en vesícula biliar y el ampular ocurren en pacientes con apertura en la segunda porción duodenal y del conducto pancreático ${ }^{2,3}$.

La coledocolitiasis tiene una incidencia del 33\% en estos pacientes y los quistes de colédoco del 10\%. Aproximadamente un tercio de los pacientes con DCBD tiene coledocolitiasis y el $10 \%$ quistes del colédoco ${ }^{16}$. La relación de $\mathrm{DCBC}$ con la unión pancreaticobiliar anormal es más frecuente en niños que presentan síntomas de dolor abdominal de forma temprana ${ }^{17}$.

Este paciente presentaba la apertura del CBCA en el estómago, según la clasificación de Choi, et al. El caso se clasificó como subtipo IV. Sin embargo, en las publicaciones no se describe ningún caso con comunicación entre las ramas intrahepáticas (como en este paciente) y es posible valorar un conducto hepático derecho que se continúa con una vía biliar común considerado como normal y un conducto hepático izquierdo que se comunica con el derecho y que se

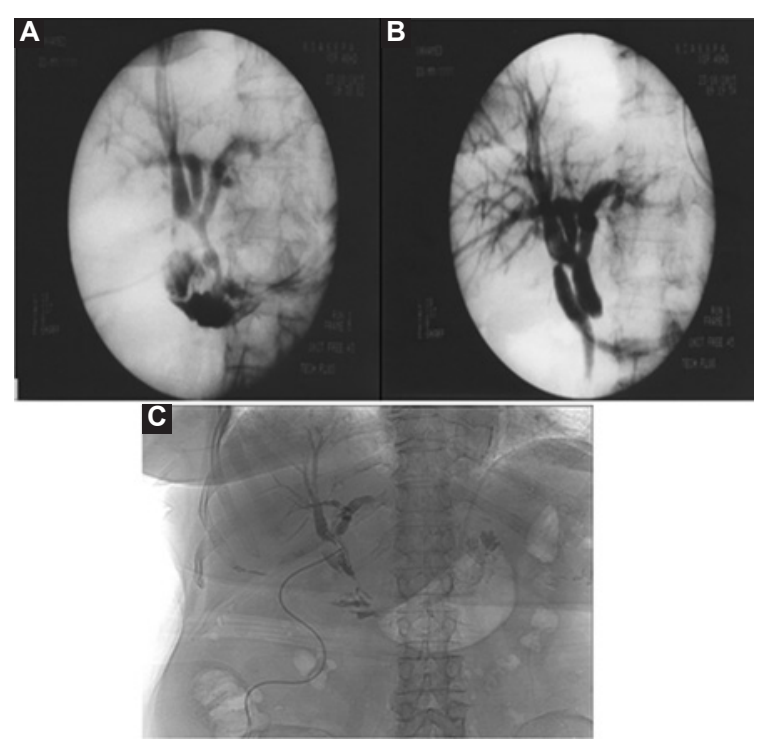

Figura 2. Colangiografía A: inicial en la que se documentan defectos de repleción en $C B C A$. B: tras tratamiento con Fogarty, antes de colocación de Kehr; se observa reperfusión de ambas vías. C: y colangiografía Trans-Kehr durante el postoporetario en la que se identifican trayectos sin defectos de repleción.

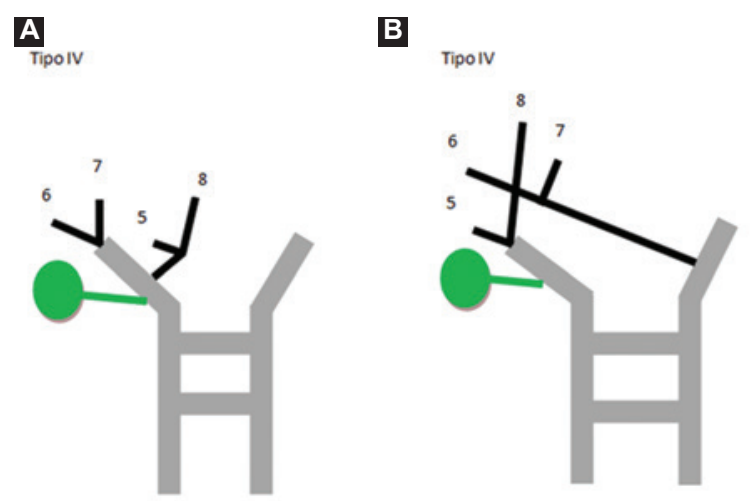

Figura 3. A: Variación de duplicación de vía biliar común tipo IV según la clasificación de Choi. B: Tipo IV con variación anatómica valorada en este paciente.

continúa con una vía biliar común accesoria. No se halló en la bibliografía ninguna descripción similar.

La incidencia de esta afección es algo superior en la mujer: 1.6:1 respecto del varón. Puede encontrarse a cualquier edad (4-80 años) y la edad media es de 46.1 años $^{10}$.

Los síntomas más frecuentes encontrados en pacientes con dicha anomalía son la inflamación del sistema biliar que se manifiesta como pancreatitis, colangitis, colecistitis, y en algunos casos aparecen absceso hepático o tumoración maligna ${ }^{10}$.

Para diagnosticar las variaciones anatómicas de las vías biliares antes de la intervención quirúrgica se usaron la CRM y la $\mathrm{CPRE}^{15}$, que permiten delinear las 
vías biliares, documentar su calibre, y valorar si existe alguna malformación o anormalidad ${ }^{5}$.

Mirizzi introdujo en 1939 la CIO en la cirugía biliar $^{18,19}$. Es una prueba invasiva que permite diagnosticar entre 6 y 10\% de variantes anatómicas biliares, como la desembocadura del conducto cístico en el conducto hepático derecho, la unión baja de ambos hepáticos o la desembocadura baja y asilada del colector posterior derecho (con incidencia del $20 \%)^{18}$. Algunos autores defienden un uso selectivo de esta prueba ${ }^{20,21}$, pero otros autores $^{19}$ prefieren la realización de forma sistemática para evitar lesiones accidentales quirúrgicas además de descartar afección litiásica. Ante la sospecha de duplicidad ductal es obligatorio el uso de $\mathrm{ClO}^{22}$, que evita lesiones innecesarias en la vía biliar $^{2}$.

El estudio exhaustivo prequirúrgico es muy importante y se realiza fundamentalmente con colangiorresonancia ${ }^{3,23}$, que indica sobre todo el lugar de confluencia de los conductos hepáticos derecho e izquierdo con una sensibilidad del $67 \%$ y una especificidad del $100 \%{ }^{19}$. De esta forma se previenen lesiones ductales accidentales $^{3}$. EI CBCA podría confundirse con un cístico y su ligadura provocaría obstrucción biliar ${ }^{3}$.

El tratamiento que se plantea ante DCBC depende de la clínica que provoque en el paciente y del tipo de apertura del $\mathrm{CBCA}^{2}$. En los casos en los que el paciente no presente cáncer no se recomienda la resección del CBCA de forma quirúrgica. En los casos sin cáncer se recomienda la resección de $\mathrm{CBCA}^{10}$ como tratamiento quirúrgico. Los pacientes con anomalías de la unión pancreatobiliar pueden necesitar la separación de la vía biliar y pancreática de forma quirúrgica para evitar así el reflujo del jugo pancreático ${ }^{3}$. Según Mason y Filipe, el reflujo biliar por sí solo no produciría patogenia tumoral, pero el reflujo pancreático y la combinación del reflujo pancreaticobiliar en un estudio animal documenta la aparición de cáncer gástrico ${ }^{24}$.

\section{Conclusiones}

La DBCB es una variante anatómica muy rara. Es importante realizar un buen estudio prequirúrgico de vías biliares con la CRM y durante la intervención quirúrgica con la $\mathrm{ClO}$ que permita prever estas circunstancias y plantear el tratamiento quirúrgico óptimo. El tratamiento a realizar depende de la clínica presentada por el paciente, así como de la presencia de afección tumoral.

\section{Financiamiento}

Los autores no recibieron financiamiento alguno.

\section{Conflicto de intereses}

No existe conflicto de intereses para la realización de dicho artículo.

\section{Agradecimientos}

Los autores agradecen a la doctora María Luisa Sánchez-Bernal por la cesión de las imágenes de anatomía patológica de la pieza quirúrgica.

\section{Responsabilidades éticas}

Protección de personas y animales. Los autores declaran que para esta investigación no se han realizado experimentos en seres humanos ni en animales.

Confidencialidad de los datos. Los autores declaran que han seguido los protocolos de su centro de trabajo sobre la publicación de datos de pacientes.

Derecho a la privacidad y consentimiento informado. Los autores han obtenido el consentimiento informado de los pacientes y/o sujetos referidos en el artículo. Este documento obra en poder del autor de correspondencia.

\section{Bibliografía}

1. Castaing D. Surgical anatomy of the biliary tract. HPB (Oxford) 2008; 10: $72-6$.

2. Djuranovic SP, Ugljesic MU, Mijalkovic NS, et al. Double common bile duct: a case report. World J Gastroenterol. 2007;13(27): 3770-3772.

3. Arora A, Sureka B, Kasana V, et al. Common bile duct duplication: the more the murkier. Saudi J Gastroenterol. 2015;21(1): 51-54.

4. Soares KC, Arnaoutakis DJ, Kamel I, el al. Choledochal cysts: presentation, clinical differentiation, and management. J Am Coll Surg. 2014; 219(6): 1167-1180.

5. D'Ippolito G, de Queiroz Rosas G, Appezzato LF, et al. Variantes anatómicas e anomalías congénitas das vías biliares e pancreáticas: análise através de colangiopancreatografía por ressonancia magnética. Rev. Imagen. 2006; 28: 33-9.

6. Chiappetta Porras L, Hernández N, Nápoli E. et al. Importancia de las variantes anatómicas de la vía biliar en la era laparoscópica. Rev. Argent. Cirug. 2000; 78: 87-95.

7. Catalano OA, Singh AH, Uppot RN, et al. Vascular and biliary variants in the liver: implications for liver surgery. Radiographics. 2008; 28: 359-78.

8. Gupta V, Chandra A. Duplication of the extrahepatic bile duct. Congenit Anom (Kyoto). 2012; 52: 176-8.

9. Krstic M, Stimec B, Krstic R, et al. EUS diagnosis of ectopic opening of the common bile duct in the duodenal bulb: a case report. World J Gastroenterol 2005;11 (32): 5068-5071.

10. Yamashita K, Oka Y, Urakami A, et al. Double common bile duct: a case report and a review of the japanese literature. Surgery. 2002; 131: 676-681.

11. Knisely AS. Biliary tract malformations. Am J Med Genet A. 2003; 122: 343-350.

12. Moore KL. Aparato digestivo. En: Embriología clínica. 4a ed. México: McGraw-Hill Interamericana, 1988:245-248.

13. Goor DA, Ebert PA. Anomalies of the biliary tree. Report of a repair of an accessory bile duct and review of the literature. Arch Surg. 1972; 104: 302-309. 
14. Saito N, Nakano A, Arase M. et al. A case of duplication of the common bile duct with anomaly of the intrahepatic bile duct. Nihon Geka Gakkai Zasshi. 1988; 89: 1296-1301.

15. Choi E, Byun JH, Park BJ, et al. Duplication of the extrahepatic bile duct with anomalous union of the pancreaticobiliary ductal system revealed by MR cholangiopancreatography. Br J Radiol. 2007; 80: 150-4.

16. Tashiro S, Imaizumi T, Ohkawa $\mathrm{H}$, et al. Pancreaticobiliary maljunction: retrospective and nationwide survey in Japan. J Hepatobiliary Pancreat Surg. 2003; 10: 345-351.

17. Tahara $\mathrm{K}$, Ishimaru $\mathrm{Y}$, Fujino J, et al. Association of extrahepatic bile duct duplication with pancreaticobiliary maljunction and congenital biliary dilatation in children: a case report and literature review. Surg Today. 2013; 43: 800-5.

18. Salas C, Pekolj J. Colangiografría intraoperatoria. Rev Argent Resid Cir. 2009; 14(1): 8-11.
19. Tolino MJ, Tartaglione AS, Sturletti CD, et al. Variedades anatómicas del árbol biliar. Implicancia quirúrgica. Int. J. Morphol. 2010; 28(4): 1235-1240.

20. Bregante M, Pirchi D, Castagneto G, et al. La colangiografía intraoperatoria selectiva en colecistitis aguda. Rev. Argent. Cirug. 91:100-4, 2006.

21. Metcalfe $\mathrm{MS}$, Ong $\mathrm{T}$, Bruening $\mathrm{MH}$, el al. Is laparoscopic intraoperative cholangiogram a matter of routine? Am J Surg. 187:475-81, 2004.

22. Otaibi W, Giang Quach G, Burke B. Double cystic duct in a septated gallbladder. Journal of Investigative Medicine High Impact Case Reports 2015: 1-2.

23. Taourel $\mathrm{P}$, Bret PM, Reinhold $\mathrm{C}$, et al. Anatomic variants of the biliary tree: diagnosis with MR cholangiopancreatography. Radiology. 1996; 199: 521-527.

24. Mason R, Filipe I. The etiology of gastric stump carcinoma in the rat. Scand J Gastroenterol. 1990; 25: 961-5. 